Int. J. Advance Soft Compu. Appl, Vol. 13, No. 3, November 2021

Print ISSN: 2710-1274, Online ISSN: 2074-8523

Copyright (C) Al-Zaytoonah University of Jordan (ZUJ)

\title{
Arrhythmia Classification Using One Dimensional Conventional Neural Network
}

\author{
Sarah kamil and Lamia AbedNoor Muhammed \\ Computer Sciences and IT College, University of Al-Qadisiyah, Iraq \\ e-mail: com.post05@qu.edu.iq \\ e-mail: Lamia.abed@qu.edu.iq
}

\begin{abstract}
Arrhythmia is a heart condition that occurs due to abnormalities in the heartbeat, which means that the heart's electrical signals do not work properly, resulting in an irregular heartbeat or rhythm and thus defeating the pumping of blood. Some cases of arrhythmia are not considered serious, while others are very dangerous, lifethreatening, and cause death in a short period of time. In the clinical routine, cardiac arrhythmia detection is performed by electrocardiogram (ECG) signals. The ECG is a significant diagnosis tool that is used to record the electrical activity of the heart, and its signals can reveal abnormal heart activity. However, because of their small amplitude and duration, visual interpretation of ECG signals is difficult. As a result, we present a significant approach for identifying arrhythmias using ECG signals. In this study, we proposed an approach based on Deep Learning (DL) technology that is a framework of nine-layer one-dimension Conventional Neural Network (1D CNN) for classifying automatically ECG signals into four cardiac conditions named: normal (N), Atrial Premature Beat (APB), Left Bundle Branch Block (LBBB), and Right Bundle Branch Block (RBBB). The practical test of this work was executed with the benchmark MIT-BIH database. We achieved an average accuracy of $99 \%$, precision of $98 \%$, recall of $96.5 \%$, specificity of 99.08\%, and an F1-score of 95.75\%. The obtained results were compared with some relevant models, and they showed that the proposed framework outperformed those models in some measures. The new approach's performance indicates its success. Also, it has been shown that deep convolutional neural networks can be used efficiently in automated detection and, therefore, cardiovascular disease protection as well as help cardiologists in medical practice by saving time and effort.
\end{abstract}

Keywords: 1-D CNN, Arrhythmia, Cardiovascular Disease, Classification, Deep learning, Electrocardiogram(ECG), MIT-BIH arrhythmia database. 


\section{Introduction}

Cardiovascular Diseases (CVDs) is the main reason of people death, in 2016 CVDs are responsible for $31 \%$ deaths of the worldwide, $85 \%$ from which happened resulting from heart attacks $\$ 555$ billion and $€ 210$ billion are the estimated annual burden of cardiovascular diseases on the American and European economy, respectively [1]. One of the most commonly types of CVDs is arrhythmia. It is an irregular heartbeat or rhythm which is one of the leading causes of mortality from cardiovascular disease (CVD)[2]. Cardiac arrhythmia is a medical state that occurs when the heart of a human pumps in an abnormal manner. Some people may have racing or fluttering heartbeats as a result of this condition. The early detection of arrhythmia can play a vital role in assisting cardiologists with decision making [3][4].

An electrocardiogram (ECG) is an electrical signal produced by the heart that is recorded by physicians in order to monitor the heart's health. An electrocardiograph with 10 electrodes placed in particular locations on the human body acquires up to twelve distinct signals, known as LEADS, in the conventional approach [5]. The ECG signals are improved by removing various types of noise and artifacts. It is a sequence of waves which consists of six distinct waveforms that may be distinguished by the letters $\mathrm{P}, \mathrm{Q}, \mathrm{R}, \mathrm{S}, \mathrm{T}$, and $\mathrm{U}[6]$.

The researchers are interested in automated arrhythmia diagnosis utilizing the ECG signal. In order to build automated monitoring systems, machine learning (ML)-based approaches such as K-nearest neighbor (KNN) algorithm, support vector machine (SVM), naïve Bayes (NB), and linear discriminant analysis (LDA) are widely used. These approaches are based on feature extraction and pattern recognition [4][7].

Deep learning (DL) is a modern branch of machine learning. The DL architecture is an extension of traditional neural network $(\mathrm{NN})$ because it involves more hidden layers between the input and output layers in order to simulate more complex nonlinear relationships [8]. Convolutional neural networks (CNNs) are a common type of DL algorithm that mimics the function of biological neural systems (such as the human brain) [9]. The CNN algorithm, which includes layers such as convolution and pooling, is run alternately until all features are extracted, after which the dropout layer, flatten layer, and finally, fully connected layer are added [10]. CNNs have great ability for features extraction that invariant to temporal/spatial and local spectral variations and that led to a large number of breakthrough results, more conspicuously in the computer vision [11].

In this paper we presented a one dimensional convolutional Neural Network (CNN) architecture for classify the dataset downloaded from MIT-BIH database (MITDB) in to four classes of arrhythmias, the classes are: Normal beat(N), Left 
Bundle Branch Block(LBBB), Right Bundle Branch Block(RBBB), and Atrial Premature Beat(ABP).

The goal of this project is to establish Computer Aided Diagnostic (CAD) system that contribute for assistant the cardiologists by presenting cost effective, time saving, and intelligent ECG arrhythmia detection.

The rest of this research is prepared as follows: Section 2 describes the literature reviews of CNNs, and section 3 presented the CNNs, arrhythmia, MITDB, and evaluation metrics used in this study. Section 4 presents the proposed model (1-D $\mathrm{CNN}$ ). section 5 describes and evaluates the results on experiment on the MITDB, and the discussion. Finally, the conclusion presents in section 6.

\section{Related Work}

In the recent years many techniques have been proposed for detect arrhythmia from ECG signals, almost of these are achieved superior performance by diagnosis various types of arrhythmias.in [12] prosed nine layer Convolutional Neural Network (CNN) for classify five classes of arrhythmia (N, S, V, F, Q), noise removal, Z-score normalization, segmentation, and R-peak are the preprocessing step that applied in this project, the performance that has been achieved with noise removal are $93.47 \%$ of accuracy, $96.01 \%$ of sensitivity, and $91.64 \%$ of specificity and the performance without noise removal accuracy of $94.03 \%$, sensitivity of $96.71 \%$, and specificity of $91.54 \%$.in [13] is proposed $16-$ layer of 1D CNN with skip connection for classify Normal , Atrial Fibrillation and other rhythm the dataset from Challenge 2017 with accuracy of $90 \%$ for Normal , 82\% for Atrial Fibrillation, and 75\% for other Rhythm.in [14] proposed 10 layer 1D Convolution Neural Network(CNN) and Long Short Term Memory(LSTM) the dataset from MIT-BIH arrhythmia database are used for classify five classes (Normal (N), Right Bundle Branch Block(RBBB), Left Bundle Branch Block(LBBB), Atrial Premature Beat (APB), Premature Ventricular Contraction(PVC)), several reprocessing steps have been implemented in this study such as R-peak detection, segmentation, and Z-score normalization, the performance that has been achieved are : $98.10 \%$ of accuracy, $97.50 \%$ of sensitivity, and $98.70 \%$ of specificity.in [15] proposed 12-layer one dimensional CNN to classify one lead individual heartbeat signal in to five classes of heart diseases (Normal beats, Left Bundle Branch Block Beat, Right Bundle Branch Block Beat, Premature Ventricular Contraction Beat, and Atrial Premature beat).The method was tested on MIT-BIH arrhythmia database and the results that has been obtained 0.977 for positive predictive, sensitivity of 0.976 , and F1-score of 0.976. in [16] two models Convolutional Neural network(CNN) and MultiLayer Perceptron (MLP) network are proposed for classification system. By MLP network two classes are classified "Arrhythmia" and "normal" with performance 88.7\% and nine classes (arrhythmia, normal sinus, second degree AV block, first degree AV block, atrial flutter, malignant ventricular, ventricular tachycardia, and ventricular bigeminy) for CNN with performance of $83.5 \%$. 


\section{Methods and Materials}

\subsection{Convolutional Neural Network (CNN)}

Convolutional Neural Network $(\mathrm{CNN})$ is one of the Artificial Neural Networks(ANNs) which is the most frequently utilized. LeCun was the originally developer of CNN in the beginning of 1990s[17]. Generally, it is a popular method for feature extraction automatically and classification of data. CNN has achieved excellent performance with problems of machine learning such as natural language processing (NLP), computer vision, and image Net and greatly results that have been obtained [18].

CNN consist of set of layers such as shown in Fig. 1; convolutional layers, fully connected layers, and pooling layers [10]. The convolutional and pooling layers are in charge of feature extraction, while fully connected layers (dense) are responsible of classification [1]. In the other side, some of these layers have parameters such as; convolutional and fully connected layers, while others with no parameters such as pooling layers. The convolution layer contains multiple filters (also known as kernels) for extracting the significant features by convolutional operation, the out from this layer are the feature maps that producing from the processes of data with the convolution kernel. each convolutional operation is followed by activation function which is nonlinear transformation [19]. Pooling layer is responsible of reducing the representation dimensionality and this in turn of effect of the model by further reducing in the computational complexity and parameters of it[20].

Convolutional neural networks(CNNs) can be in different forms; 1-D, 2-D, and 3-D due to the dimension of input data. 2-D CNNs are applied with two data dimensions' type such as videos and images, 1-D CNNs which are modification version of 2-D. 1-D CNNs have different applications of one dimension especially with signal processing such as; structural damage detection, structural health monitoring, and early stage detection of arrhythmia in ECG beats.

The1-D CNNs become really celebrated with the state-of-the-art performance in record time [21]. Many reasons that make 1-D CNNs are more preferable and advantageous in applications of signal processing, lower computational, less complexity of 1-D CNN against 2-D CNN under the same conditions, i.e., the same hyper parameters, network, and configuration, the configuration of 1-D $\mathrm{CNN}$ is really shallow that make the train and implement of the networks are much easier to done. The implementation of 1-D CNNs doesn't require special hardware as their 2-D counterparts, lastly, 1-D CNNs are well- suited for applications with mini- cost and real- time in particular on mobile [21]. 


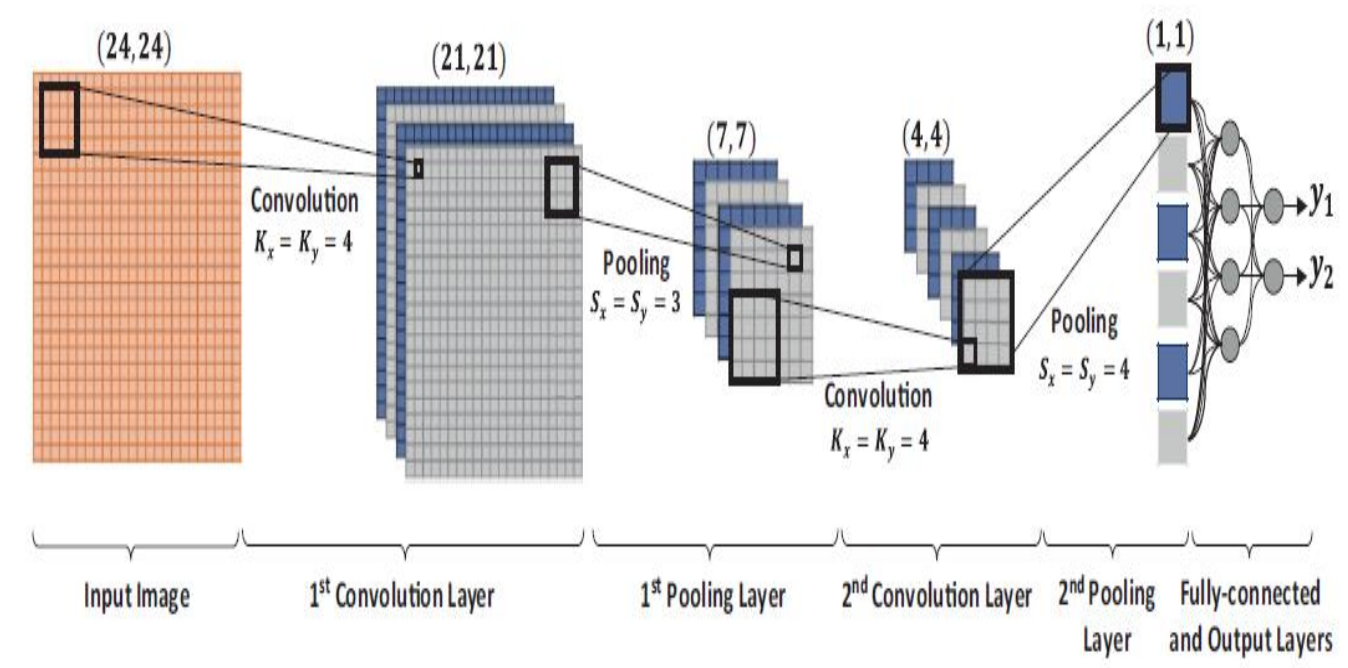

Fig. 1 Sample of 2-D CNN architecture

\subsection{Arrhythmia}

Arrhythmia is the abnormality or irregularity in the rhythm of heartbeats. For the healthy human, the range of normal heart rate is between $60-100 \mathrm{Bpm}$. With arrhythmia, a patient can feel their heartbeat racing, fluttering or skipping more or like palpitation. Arrhythmia can be categorized in three major types according to the range of heart rate, these types are: tachycardia, bradycardia, and extra-beat.

Tachycardia arrhythmia refers to heart beat above the normal heart rate such as fibrillation and flutter. Bradycardia arrhythmia refers to heartbeat below the normal heart rate such as Left and Right Bundle Branch Block. Extra heartbeat refers to the forceful beat or irregularity of the heartbeat rate such as Atrial premature beat [21].

To detect the type of arrhythmia, ECG signal features are tracked. However, it consists of waves, intervals, and segments. The normal ECG signal as shown in the Fig. 2, while different types of disease according to arrhythmia have different shape and size of its wave. R-peaks have the highest amplitudes that making them the easiest wave for disclosure and excellent reference of points for eventuality detections [22]. 


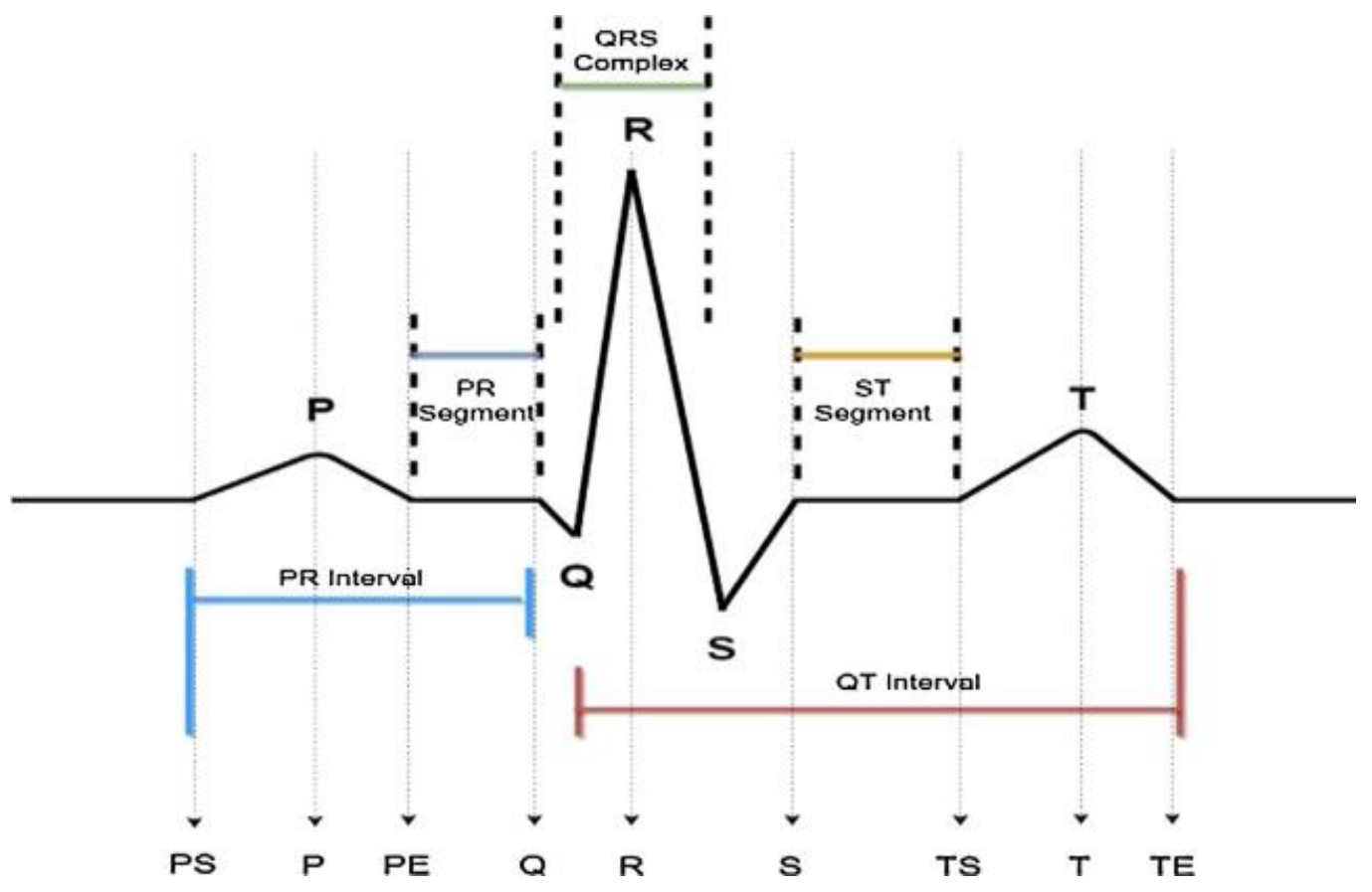

Fig. 2 The normal ECG signal [23].

arrhythmia can be classified in to two main types: morphological arrhythmia and rhythmic arrhythmias. morphological arrhythmia is formatted by only one an irregular Heartbeat such as: Right and Left Bundle Branch Block beat (RBBB, LBBB) while Rhythmic arrhythmias are formed by multiple of irregular heartbeats such as Atrial Fibrillation(AF)[24]. according to AAMI the heartbeats have been classified in to five types: $N$ (Normal Beat), $S$ (Supraventricular Ectopic Beats(SVEB)), V (Ventricular Ectopic Beat(VEB)), Fusion Beat(F), and $\mathrm{Q}$ (un classified beats, as well as paced beats) [23]. Fig. 2 represent several types of cardiac arrhythmias.

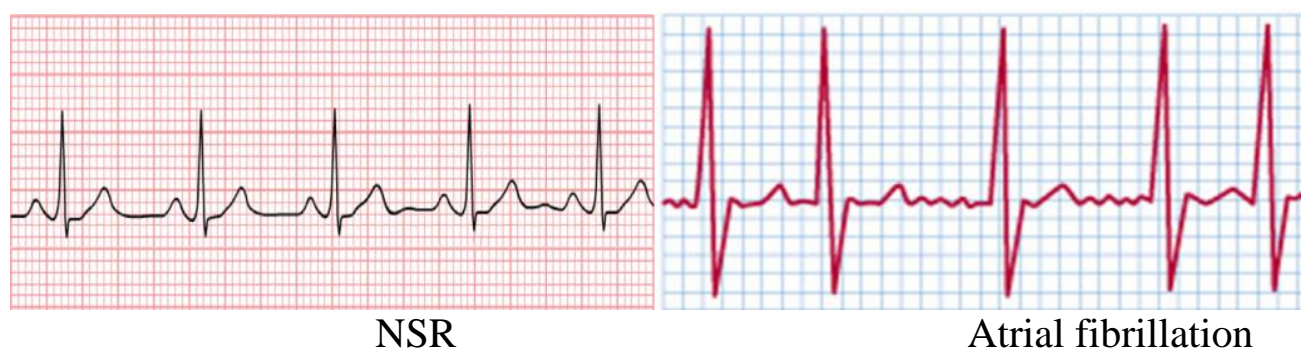




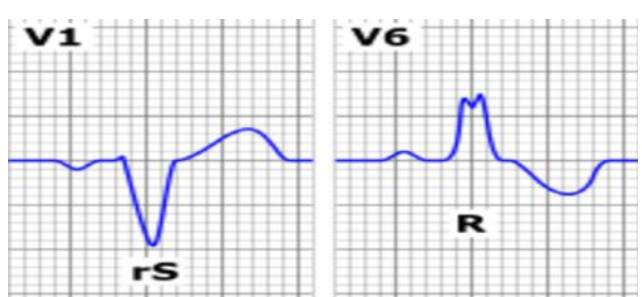

LBBB
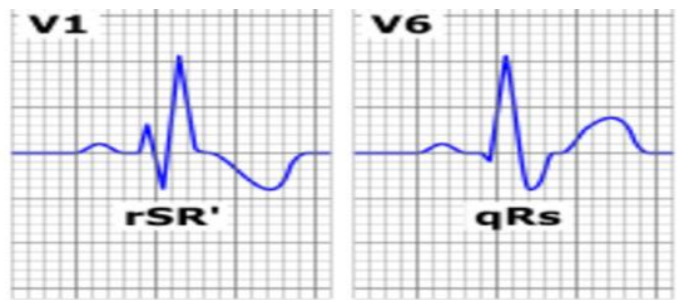

RBBB

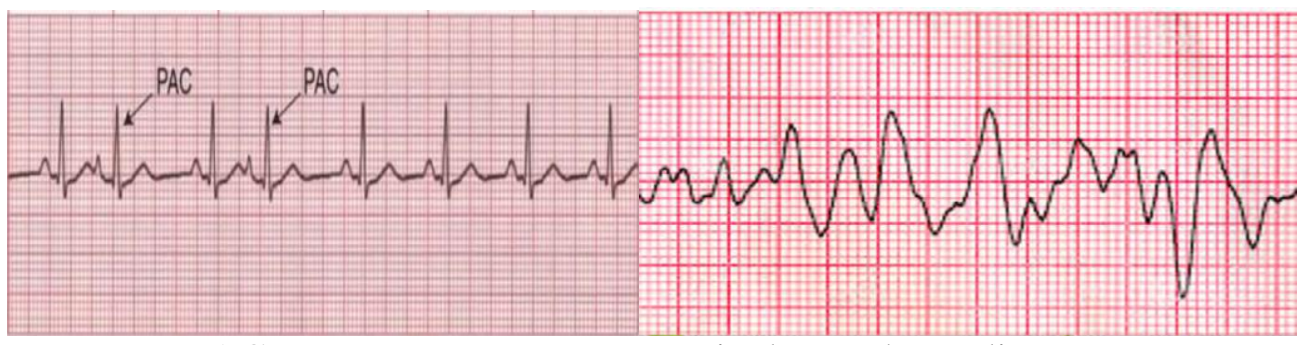

PAC

Ventricular Tachycardia

Fig. 3 Types of arrhythmia [23]

\subsection{Evaluation metrics}

The performance of the classification model can be evaluated through different measures. However, the actual class values are available for testing data, the predicate value for this class would be resulted from the tested model. Then, compare the predicate values and actual values and construct the confusion matrix as shown in Table 1.

Table 1: Confusion Matrix for Binary Classification

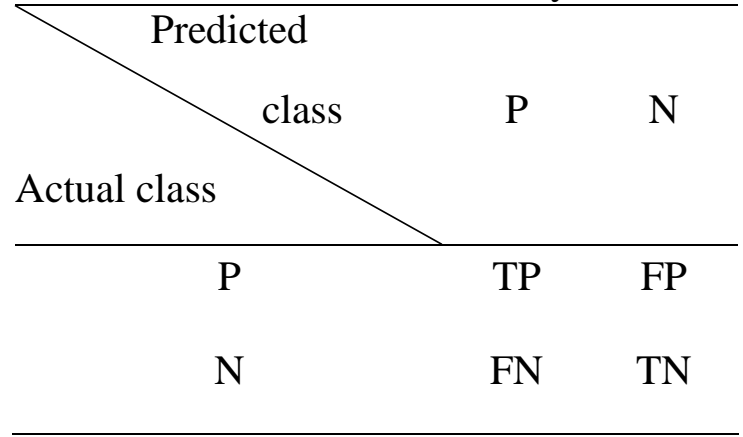

The confusion matrix has four states according to the correct or incorrect prediction [1], the possible states are:

- True Positive(TP): correct prediction of positive class

- False Positive(FP): incorrect prediction of positive class

- True Negative(TN): correct prediction of negative class

- False Negative(FN): incorrect prediction of negative class 
Different metrics can be extracted from confusion matrix; accuracy, specificity, sensitivity, precision, and F1. The average accuracy is a total of accuracies for all classes divided by number of classes. the classification accuracy is defined as the sum of true positive and true negative (the total number of correctly classified cases) divided by full amount of cases.

Accuracy $=\frac{T P+T N}{T P+T N+F P+F N}$

The specificity (Sp), The sensitivity (Sen) and precision(Pre) were calculated as:

$$
\begin{aligned}
& S p=\frac{T N}{T N+F P} \\
& \text { Sen }=\frac{T P}{T P+F N} \\
& \text { Pre }=\frac{T P}{T P+F P}
\end{aligned}
$$

Precision(Pre) and recall(Sen) were using for calculate F1-score as:

$F 1-$ score $=2 \times\left(\frac{\text { Pre } \times \text { Sen }}{\text { Pre }+ \text { Sen }}\right)$

\subsection{MIT-BIH arrhythmia database (MITDB)}

MIT-BIH arrhythmia database is a public data set. It was captured at Laboratory for Computational Physiology (LCP) at the Harvard-MIT Division of Health Sciences and Technology, is a center for research into subjects such as cardiac arrhythmia detection; heart rate variability; compression, transmission, storage, and retrieval of physiologic signals; cardiovascular and pulmonary dynamics; and medical decision support for intensive care.

The datasets contain 48 recordings of two channels of ECG signals with half hour duration from 47 subjects between (1975-1979). The subjects were 22 women age 23 - 89 and 25 men age 32 -89. In addition to the recordings, this database including timing information and an annotation of the information about the beat class and that verified by two independent experts [25].

\section{The Proposed Model}

The proposed model is 1-D convolution neural network, architecture of the model is shown in the Table 2, which illustrated the comprehensive presentation of the layers in the proposed model. The architecture of the proposed 1-D CNN classification has well defined with 9 layers:

- Three 1-D convolutional layers.

- One Maxpooling 1D layer.

- One Dropout layer.

- One Flatten layer.

- Three Fully connected layers(Dense). 
Table 2: The hierarchy details of the proposed CNN model

\begin{tabular}{lllllll}
\hline Layers & Type & $\begin{array}{l}\text { Filter } \\
\text { size }\end{array}$ & stride & Kernel & $\begin{array}{l}\text { Activation } \\
\text { function }\end{array}$ & Parameters \\
\hline Layer1 & Conv1D & 5 & 1 & 32 & ReLU & 192 \\
Layer2 & Conv1D & 5 & 1 & 64 & ReLU & 10304 \\
Layer3 & Conv1D & 5 & 1 & 128 & ReLU & 41088 \\
Layer4 & MaxPooling1D & 5 & 2 & - & - & - \\
Layer5 & Dropout & 0.5 & & - & - & - \\
Layer6 & Flatten & - & & - & - & - \\
Layer7 & Fully & - & & 512 & ReLU & 4194816 \\
& connected & & & & & 525312 \\
Layer8 & Fully & - & & 1024 & ReLU & \\
& connected & & & & & Softmax \\
Layer9 & Output layer & - & & 4 & & 4100 \\
\hline
\end{tabular}

\section{$5 \quad$ Experimental results and Discussion}

the dataset that used for evaluate the model has been downloaded from MIT-BIH arrhythmia database. the dataset has been taken from a single0 lead which is Modified Limb Lead(MLII). for further preprocessing. In-max normalization is applied for the data to make it in one scale size between 1 and 0 . R-peaks detection are applied for detect the peaks for each record as shown in the figure3. the sequences are deposition by select 63 samples before the peaks and 64 samples after the peaks to constitute one sequence with 128 samples. after forming the dataset in to sequences, each sequence is labeled with one of the classes "N", "LBBB", "RBBB" and "APB". for the experiments of classify of ECG signal in to four classes of arrhythmia, 39091 sequences are extracted and it has been segmented among the four classes as follow:

- Normal class of 30777

- Left Bundle Branch Block of 3798

- Right Bundle Branch Block of 3144

- Atrial Premature Beat of 1372

the percentage of arrhythmia four classes illustrated in Fig. 4.

The overall process as shown in Fig. 5 can be summarized in the four basic steps, the steps are: download one dimensional data from MIT-BIH arrhythmia database, preprocessing the data by set of methods such as normalization and R-peaks detection, feature extraction by 1-D CNN model, and classification four ECG signal (normal class and three classes of arrhythmias). Fig. 6 illustrates R-peaks detection along one minute of the record 100. 


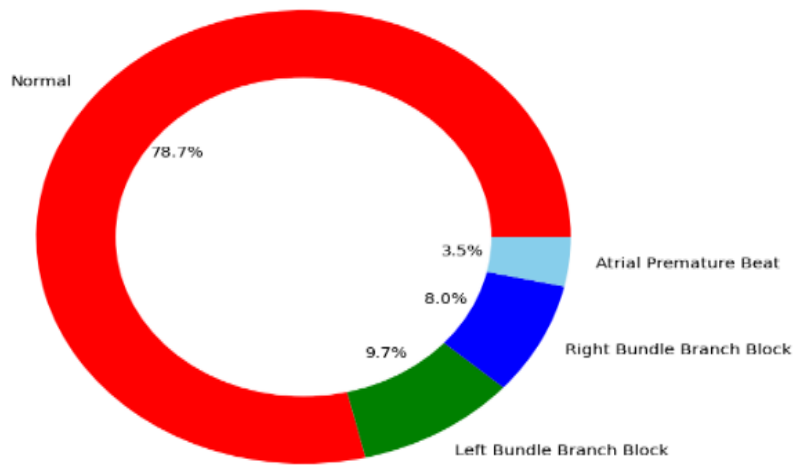

Fig. 4 The percentage of the arrhythmia four classes

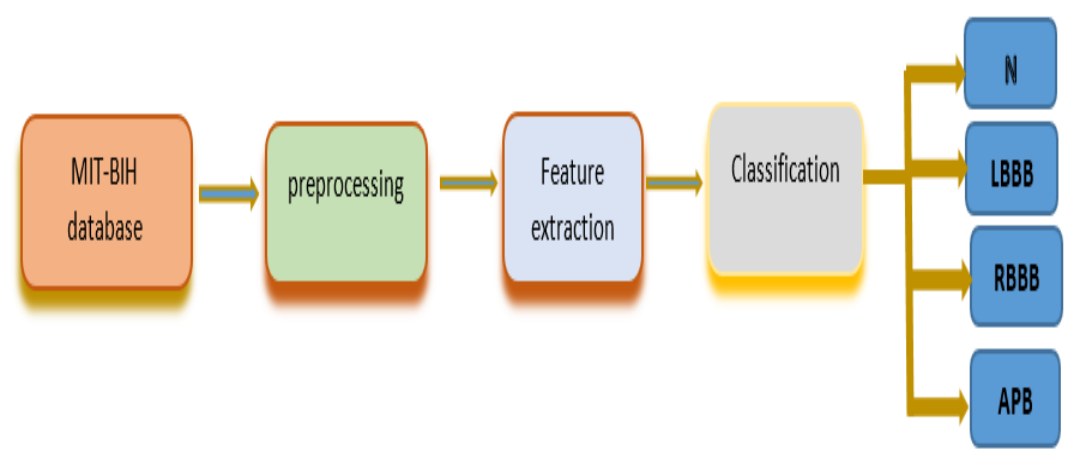

Fig. 5 Block diagram of the steps of ECG signal classification

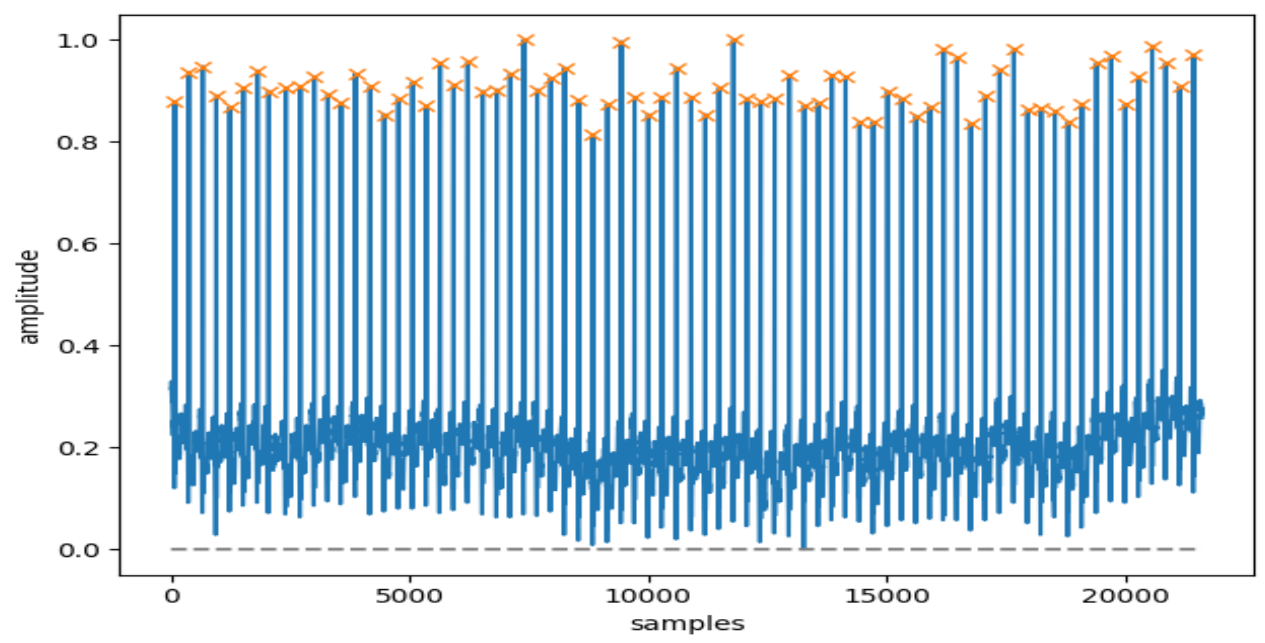

Fig. 6 Detection of R-peaks in ECG signals 
The dataset that used are divided in to training set and testing set. $80 \%$ and $20 \%$ are the percentage of the training set and the testing set of the data respectively as shown in Fig. 7.

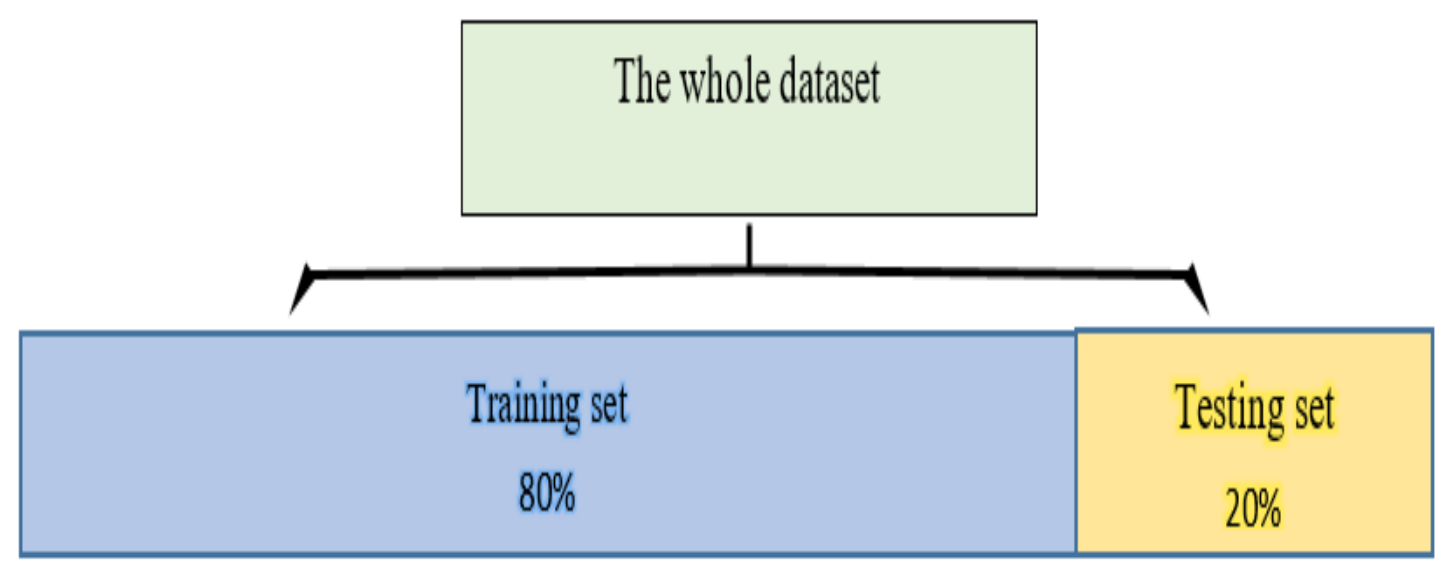

Fig. 7 The percentage of the training and testing dataset

After training the model, it was tested with the test data. The results that has been obtained by using more than one metrics such as: precision, Recall, F1-score, specificity, and average accuracy as shown in the Table 3

Table 3: Precision, recall, F1-score, specificity, and average accuracy for the proposed CNN model.

\begin{tabular}{llllll}
\hline Metric & $\mathrm{N}$ & LBBB & RBBB & APB & Overall \\
\hline Precision & $99 \%$ & $100 \%$ & $100 \%$ & $93 \%$ & $98 \%$ \\
Recall & $100 \%$ & $100 \%$ & $99 \%$ & $79 \%$ & $96.5 \%$ \\
F1-score & $99 \%$ & $100 \%$ & $99 \%$ & $85 \%$ & $95.75 \%$ \\
Specificity & $96.61 \%$ & $99.95 \%$ & $99.97 \%$ & $99.78 \%$ & $99.08 \%$ \\
Average & & & & & $99 \%$ \\
accuracy & & & & & \\
\hline
\end{tabular}

the ROC-Curve for the four classes (N, LBBB, RBBB, and APB) are represented in Fig. 8. 


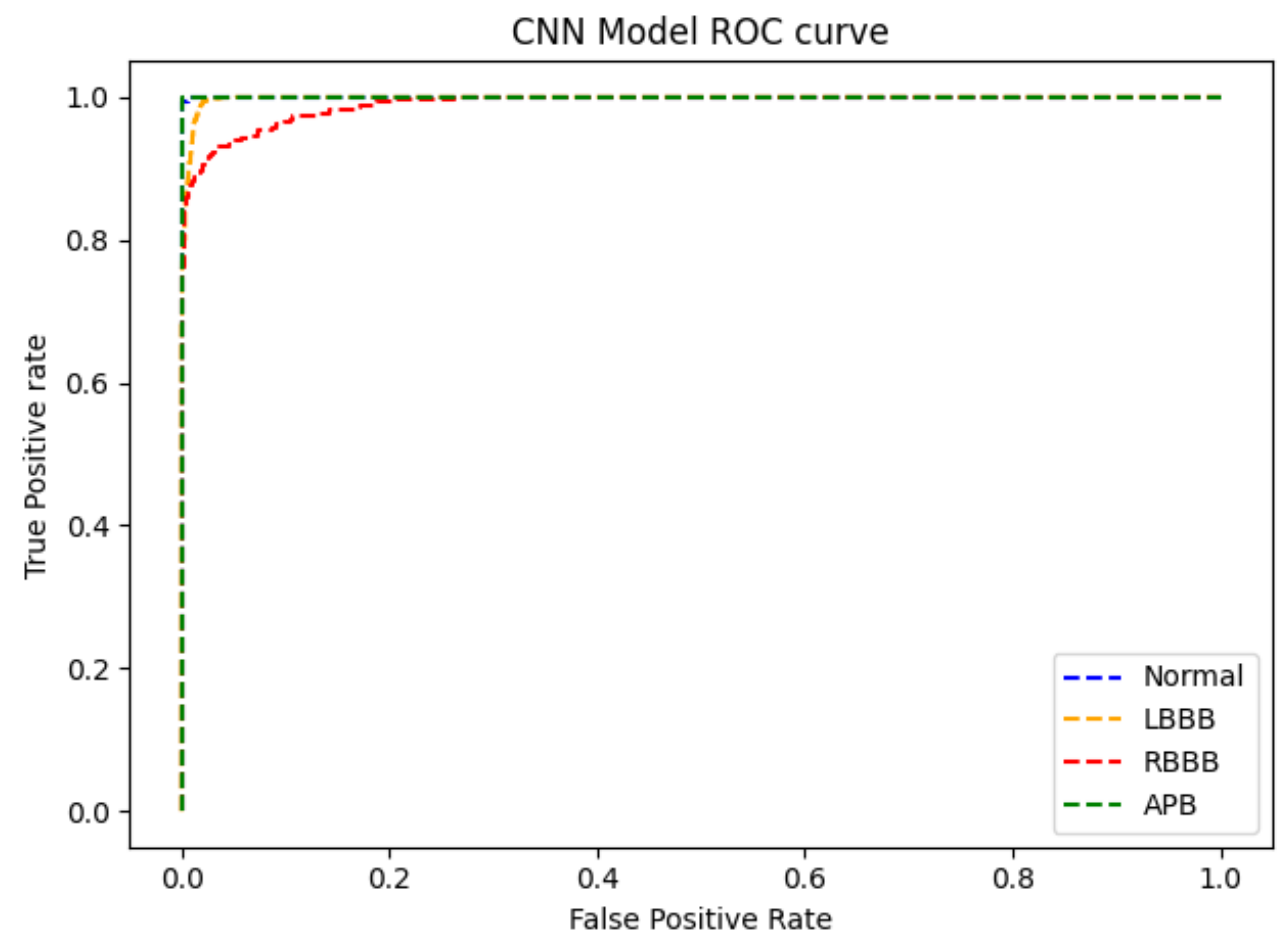

Fig. 8. Roc-Curve for four classes of arrhythmia

The loss, accuracy, validation-loss, and validation- accuracy have been shown in the Fig. 9.

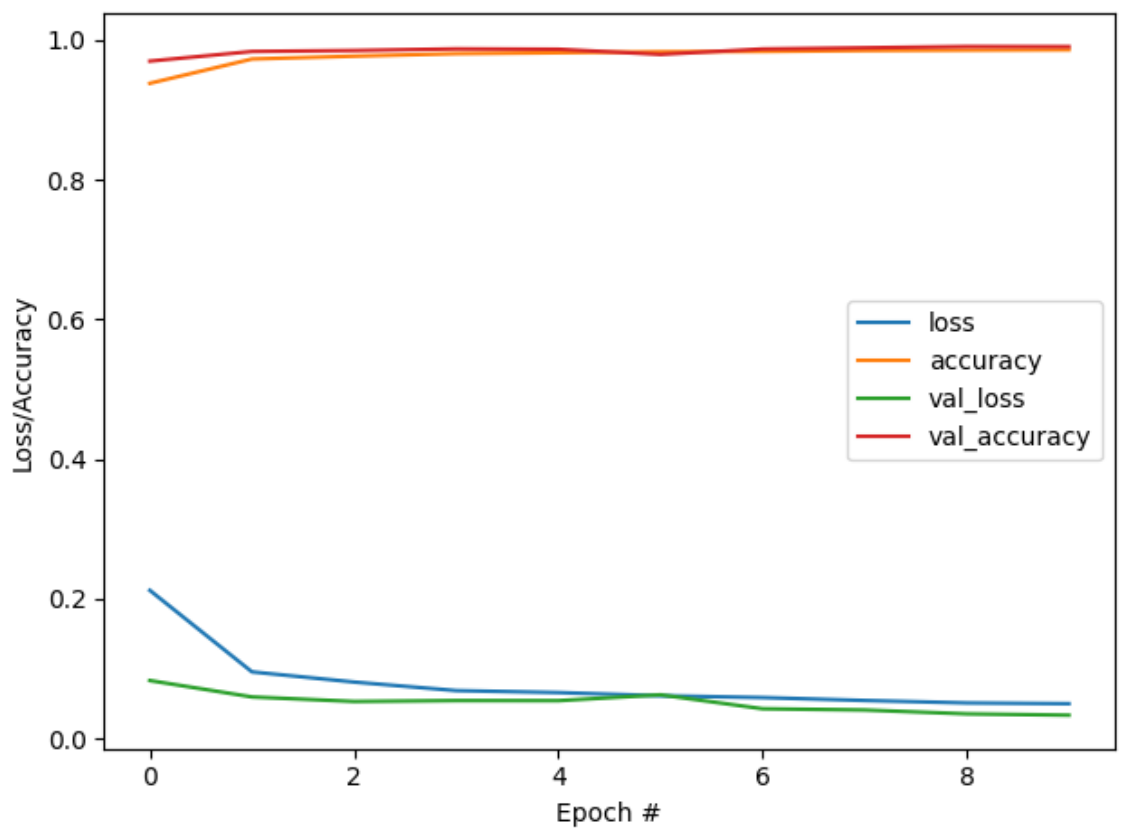

Fig. 9: Performance metrics of the proposed model 
Convolutional Neural Networks(CNNs) have significant facility to extract the distinct features that relatively invariant to temporal variations and local spectral. ECG data that has been obtained from MIT-BIH arrhythmia database has get preprocessed by normalizing it and detect the R-peaks, finally, the sequences are forming and the labels are assigned. after the previous steps have been completed, we design, training, and testing the network that exploiting the various features as inputs. The proposed model has get evaluated for classification of ECG signal in to four distinct cardiac conditions.

The batch size of the data and the epochs have been greatly affected the accuracy and the behavior of the model. So, we chosen the batch size at 20 and epochs 10 . Although, it is very little, it is get great accuracy of 99\%. The proposed CNN algorithm took approximate 420.1 seconds to complete the training epochs. The detailed performance of the proposed CNN model is presented using Confusion matrices for the four classes. The cases that classified correctly are the elements in the diagonal axis, whereas an incorrect case are presented in the anything else the diagonal. The confusion matrixes of the proposed model with and without normalization are illustrated in Fig.10 and Fig.11, respectively.

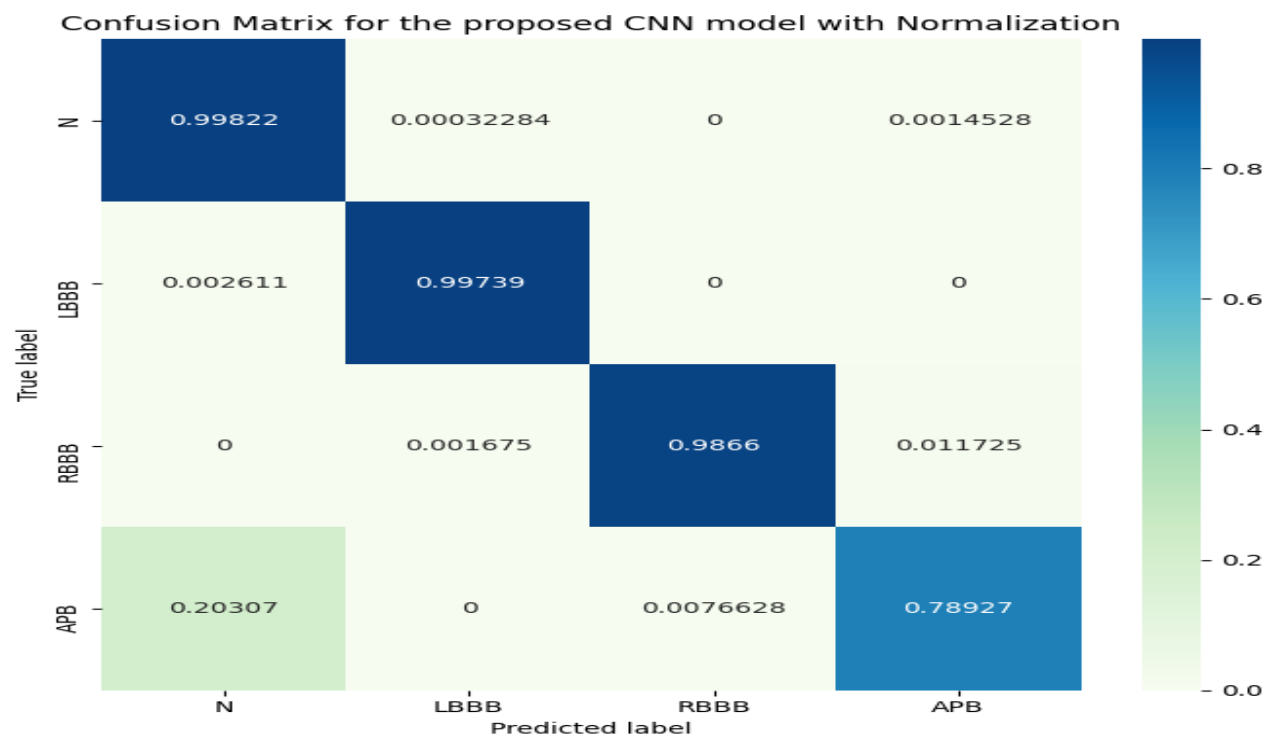

Fig. 10: Confusion matrix of the CNN model with normalization 


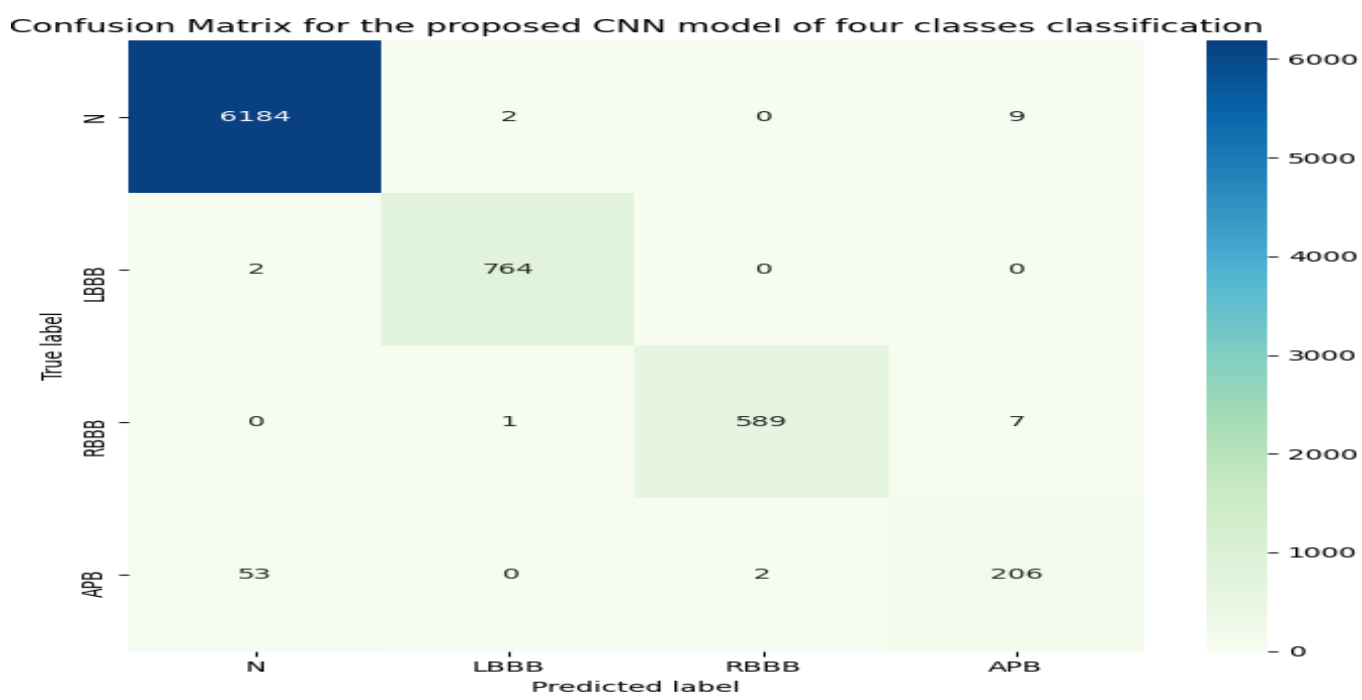

Fig. 11: Confusion matrix of the CNN model

To give more accurate view about proposed model, comparison has been accomplished with the close works as shown in Table 4, summarizes evaluation of the performance of the proposed CNN model with other techniques that operates on arrhythmias classification using ECG signals.

It should be notable that different techniques have been used 1-D data for arrhythmia classification. Among these techniques, [26] proposed eleven layer 1D CNN for classify only two classes with average accuracy $95.22 \%$, this study is lower than the proposed model in the number of classes that has been classified and the performance of the model. In [16] the researchers presented a classification system of CVDs using CNN network and MLP (Multi-Layer Perceptron) network, two classes (normal and arrhythmia) has been used in the MLP network and nine classes has been used in the CNN network. This study showed lower performance than the proposed model in both using CNN network and MLP network, i.e.,83.5 and 88.7 respectively. [13][27] get models that classify only three classes with lower performance than the proposed model that classify four classes, the proposed model outperformed these models in the number of classes than have been classified and the performance that has been achieved. The study [28] proposed 1-D CNN for detecting five classes, this model has not been able to get high performance. This study [14] has applied CNN and LSTM (Long Short Term Memory) for classifying five classes by comparing with the proposed model, the proposed architecture has lower complicating and it has attained higher performance in both accuracy and specificity metrics. 
Table 4: Comparison of the proposed CNN model with recent arrhythmia classification techniques.

\begin{tabular}{|c|c|c|c|c|c|c|c|}
\hline $\begin{array}{l}\text { Researcher } \\
\text { and year }\end{array}$ & Classes & Acc\% & Sen $\%$ & $\begin{array}{l}\text { Spec } \\
\%\end{array}$ & Pre\% & Model & database \\
\hline $\begin{array}{l}\text { Acharya, et al., } \\
\text { 2017[26] }\end{array}$ & 2 & 95.22 & & & - & $\begin{array}{l}\text { 11-layer } \\
\text { 1-D CNN }\end{array}$ & MITDB \\
\hline $\begin{array}{l}\text { Savalia } \quad \& \\
\text { Emamian, } \\
\text { 2018[16] }\end{array}$ & $\begin{array}{l}9(\mathrm{CNN}) \\
2(\mathrm{MLP})\end{array}$ & $\begin{array}{l}83.5 \\
\text { CNN } \\
88.7 \\
\text { MLP }\end{array}$ & & & & $\begin{array}{l}\text { 5-layer } \\
1 \text {-D CNN }\end{array}$ & $\begin{array}{l}\text { MITDB\& } \\
\text { kaggar }\end{array}$ \\
\hline $\begin{array}{l}\text { Zubair et al., } \\
2016[28]\end{array}$ & 5 & 92.7 & & & & $\begin{array}{l}\text { 4-layer } \\
\text { 1-D CNN }\end{array}$ & MITDB \\
\hline $\begin{array}{l}\text { Xiong et al., } \\
2018[13]\end{array}$ & 3 & 82 & - & - & - & $\begin{array}{l}\text { 21-layer } \\
\text { 1-D CRNN }\end{array}$ & $\begin{array}{l}\text { Challenge } \\
2017\end{array}$ \\
\hline $\begin{array}{l}\text { Oh et al., } \\
2018[14]\end{array}$ & 5 & 98.10 & 97.50 & 98.70 & & $\begin{array}{l}\text { CNN+LST } \\
\mathrm{M}\end{array}$ & MITDB \\
\hline $\begin{array}{l}\text { Avanzato \& } \\
\text { Beritelli, } \\
\text { 2020[27] }\end{array}$ & 3 & 98.33 & & & & $\begin{array}{l}\text { 5-layer } \\
1 \text {-D CNN }\end{array}$ & MITDB \\
\hline $\begin{array}{l}\text { Proposed CNN } \\
\text { model }\end{array}$ & 4 & 99.00 & 94.5 & 99.08 & 98 & $\begin{array}{l}\text { 9-layer } \\
\text { 1-D CNN }\end{array}$ & MITDB \\
\hline
\end{tabular}

Proposed model achieved $99 \%$ average accuracy, 99.08\% specificity, 94.5\% sensitivity, and $98 \%$ positive predictive value (precision). These values are excellent when they would be compared with the recent techniques that utilized of $\mathrm{CNN}$ algorithm for the equivalent automatic arrhythmia classification.

\section{Conclusion}

In this paper, we proposed a 1-D CNN classification model for classifying cardiac arrhythmias automatically utilizing ECG signals. an accurate classification of ECG signals has been helpful with extreme degree for diagnosis and prevention of CVDs. The proposed CNN classification algorithm which using 1-d time series data, can classify four classes of arrhythmia, that is, Normal, LBBB, RBBB, and APB, and it is accomplished $99 \%$ average accuracy, 99.08\% specificity, $94.5 \%$ sensitivity, and $98 \%$ positive predictive value (precision). These results show that the 1-D CNN model is a reliable technique for detection and classification of CVDs.

Through this work, it was found there is need for more test with ECG signals from local medical centers, so it may be performed as a future work.

\section{References}

[1] Z. Ebrahimi, M. Loni, M. Daneshtalab, and A. Gharehbaghi, (2020). A review on deep learning methods for ECG arrhythmia classification. Expert Syst. with Appl. X (7), 100033.

[2] T. Wang, C. Lu, Y. Sun, M. Yang, C. Liu, and C. Ou, (2021). Automatic ECG 
classification using continuous wavelet transform and convolutional neural network. Entropy, 23(1), 1-13.

[3] Z. F. M. Apandi, R. Ikeura, and S. Hayakawa, (2018). Arrhythmia Detection Using MITBIH Dataset: A Review.2018 Int. Conf. Comput. Approach Smart Syst. Des. Appl. ICASSDA 2018, no. August.

[4] M. Thirugnanam and M. S. Pasupuleti, (2021), Cardiomyopathy -induced arrhythmia classification and pre-fall alert generation using Convolutional Neural Network and Long Short-Term Memory model, Evol. Intell., 14(2), 789-799.

[5] J. Ferretti, V. Randazzo, G. Cirrincione, and E. Pasero, (2021). 1-D Convolutional Neural Network for ECG Arrhythmia Classification,Smart Innov. Syst. Technol., 184, 269-279.

[6] S. Nurmaini, P. R. Umi, R. M. Naufal, and A. Gani, (2018). Cardiac arrhythmias classification using Deep Neural Networks and principle component analysis algorithm, Int. J. Adv. Soft Comput. its Appl., 10(2), 14-32.

[7] Y. Li and W. Cui, (2019). Identifying the mislabeled training samples of ECG signals using machine learning, Biomed. Signal Process. Control,47, 168-176.

[8] H. Mohsen, E.-S. A. El-Dahshan, E.-S. M. El-Horbaty, and A.-B. M. Salem, (2018). Classification using deep learning neural networks for brain tumors, Futur. Comput. Informatics J., 3(1), 68-71.

[9] G. Petmezas et al., (2021). Automated Atrial Fibrillation Detection using a Hybrid CNNLSTM Network on Imbalanced ECG Datasets, Biomed. Signal Process. Control, 63(March 2020), 102194.

[10] G. S. Nandini, A. P. S. Kumar, and C. K,(2021). Dropout technique for image classification based on extreme learning machine. Glob. Transitions Proc., 2(1), 111-116.

[11] M. Zihlmann, D. Perekrestenko, and M. Tschannen,(2017). Convolutional recurrent neural networks for electrocardiogram classification. Comput. Cardiol. (2010)., 44, 1-4.

[12] U. R. Acharya, H. Fujita, O. S. Lih, Y. Hagiwara, J. H. Tan, and M. Adam,(2017). Automated detection of arrhythmias using different intervals of tachycardia ECG segments with convolutional neural network. Inf. Sci. (Ny)., 405, 81-90.

[13] Z. Xiong, M. P. Nash, E. Cheng, V. V. Fedorov, M. K. Stiles, and J. Zhao,(2018). ECG signal classification for the detection of cardiac arrhythmias using a convolutional recurrent neural network. Physiol. Meas., 39(9), 1-14.

[14] S. L. Oh, E. Y. K. Ng, R. S. Tan, and U. R. Acharya, (2018).Automated diagnosis of arrhythmia using combination of CNN and LSTM techniques with variable length heart beats. Comput. Biol. Med., 102, 278-287.

[15] W. Zhang, L. Yu, L. Ye, W. Zhuang, and F. Ma, (2018). ECG Signal Classification with Deep Learning for Heart Disease Identification. Int. Conf. Big Data Artif. Intell. BDAI 2018, no. June, 47-51.

[16] S. Savalia and V. Emamian, (2018). Cardiac arrhythmia classification by multi-layer perceptron and convolution neural networks, Bioengineering, vol. 5, no. 2.

[17] C. Chen, Z. Hua, R. Zhang, G. Liu, and W. Wen, (2020). Automated arrhythmia classification based on a combination network of CNN and LSTM, Biomed. Signal Process. Control, 57, 101819.

[18] S. Albawi, T. A. Mohammed, and S. Al-Zawi, (2018). Understanding of a convolutional neural network. Proc. 2017 Int. Conf. Eng. Technol. ICET 2017, 2018-Janua, 1-6.

[19] X. Liu, Q. Zhou, J. Zhao, H. Shen, and X. Xiong,(2019). Fault diagnosis of rotating machinery under noisy environment conditions based on a 1-D convolutional autoencoder and 1-D convolutional neural network, Sensors (Switzerland), 19(4),1-13 .

[20] K. O'Shea and R. Nash, (2015). An Introduction to Convolutional Neural Networks, [Online]. Available,1-11

[21] S. Kiranyaz, O. Avci, O. Abdeljaber, T. Ince, M. Gabbouj, and D. J. Inman, (2021).1D convolutional neural networks and applications: A survey. Mech. Syst. Signal Process., 151, 107398.

[22] S. S and A. V,(2019). Early Detection Of Life-Threatening Cardiac Arrhythmias Using Deep Learning Techniques. Curr. Signal Transduct. Ther., 14, 1-12. 
[23] S. Sahoo, M. Dash, S. Behera, and S. Sabut, (2020). Machine Learning Approach to Detect Cardiac Arrhythmias in ECG Signals: A Survey. Irbm, 41(4), 185-194.

[24] W. Sun, N. Zeng, and Y. He, (2019).Morphological Arrhythmia Automated Diagnosis Method Using Gray-Level Co-Occurrence Matrix Enhanced Convolutional Neural Network, IEEE Access, 7(c), 67123-67129.

[25] G. B. Moody and R. G. Mark,(2001).The impact of the MIT-BIH arrhythmia database. IEEE Eng. Med. Biol. Mag., 20(3), 45-50.

[26] U. R. Acharya, H. Fujita, S. L. Oh, Y. Hagiwara, J. H. Tan, and M. Adam,(2017).Application of deep convolutional neural network for automated detection of myocardial infarction using ECG signals. Inf. Sci. (Ny)., 415-416, 190-198.

[27] R. Avanzato and F. Beritelli,(2020).Automatic ecg diagnosis using convolutional neural network. Electron., 9(6), 1-14.

[28] M. Zubair, J. Kim, and C. Yoon,(2016).An automated ECG beat classification system using convolutional neural networks. 2016 6th Int. Conf. IT Converg. Secur. ICITCS 2016.

\section{Notes on contributors}

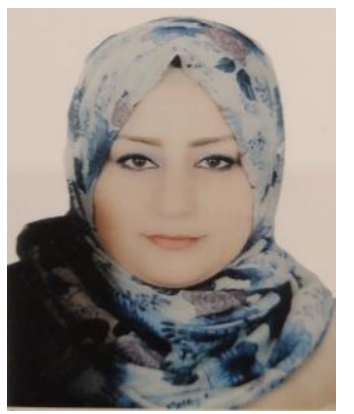

Sarah kamil is a MA. Student at Computer sciences, University of Al-Qadisiyah, Iraq. She works for more than 10 years as lecturer in secondary school. Her research interests in application of Deep learning in diagnosis heart disease. She aims to achieve good contribution in classification arrhythmia.

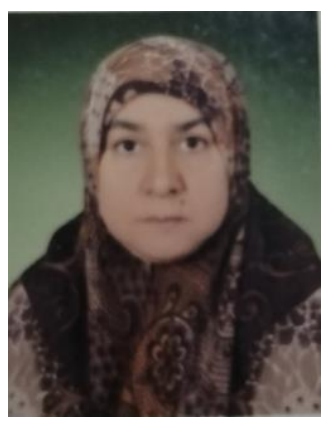

Lamia AbedNoor Muhammed is an Ass. Professor at the Department of Computer sciences, Al-Qadisiyah University, Iraq. .Her main teaching in under-graduate and post-graduate and research interests include with subjects; Artificial Intelligent, Machine learning, Data mining, and Information system. 